\title{
Flavonoids quantification in Acer negundo L., extracts by HPLC analysis
}

\author{
Salgado-Garciglia, Rafael ${ }^{1}$; Hernández-García, Alejandra ${ }^{1}$; Montiel-Montoya, Jorge ${ }^{2}$; \\ Valdez-Morales, Maribel ${ }^{3}$; López-Valdez, Luis Germán ${ }^{4}$; Herrera-Cabrera, Braulio Edgar ${ }^{5}$; \\ Zaragoza-Martínez, Fabiola $^{6}$; Lucho-Constantino, Gonzalo Guillermo ${ }^{7}$; Barrales-Cureño, Hebert Jair ${ }^{{ }^{*}}$ \\ ${ }^{1}$ Universidad Michoacana de San Nicolás de Hidalgo. \\ ${ }^{2}$ Instituro Politécnico Nacional, Unidad Sinaloa, centro Interdisciplinario de Investigación Integral Regional. \\ ${ }^{3}$ Consejo Nacional de Ciencia y Tecnología (CONACyT). Centro Interdisciplinario de Investigación para el \\ Desarrollo Integral Regional. \\ ${ }_{5}^{4}$ Universidad Autónoma Chapingo. \\ ${ }^{5}$ Colegio de Postgraduados, Campus Puebla. \\ ${ }^{6}$ Centro de Investigación y Estudios Avanzados del IPN. \\ ${ }^{7}$ Universidad Tecnológica de Gutiérrez Zamora. \\ *Corresponding author: hebert.jair@uiep.edu.mx
}

Citation: Salgado-Garciglia, R., HernándezGarcía, A., Montiel-Montoya, J., ValdezMorales, M., López-Valdez, L. G., HerreraCabrera, B. E., Zaragoza-Martínez, F., Lucho- Constantino, G. G., \& BarralesCureño, H.J. (2021). Flavonoids quantification in Acer negundo L., extracts by hplc analysis. Agro Productividad, 14(\#). https://doi.org/10.32854/ agrop.v14i7.1953

Editor in Chief: Dr. Jorge Cadena Iñiguez

Estimated publication date: August 2021

This work is licensed under a Creative Commons Attribution-Non-Commercial 4.0 International license.

\begin{abstract}
Objective: The identify and quantify, by high performance liquid chromatography, flavonoids from leaf and stem extracts of Acer negundo.

Design/methodology/approach: Ethanolic extracts of Acer negundo were analysed with high performance liquid chromatography to quantify and identify their major antioxidant flavonoids.

Results: Leaf extracts had high concentrations of rutin $(34.19 \mu \mathrm{g} / \mathrm{mL})$ and catechin $(33.97 \mu \mathrm{g} / \mathrm{mL})$, intermediate concentrations of apigenin $(19.05 \mu \mathrm{g} / \mathrm{mL})$, gallic acid $(19.04 \mu \mathrm{g} / \mathrm{mL})$, ferulic acid $(17.2 \mu \mathrm{g} / \mathrm{mL})$ and 2.5 dihydroxybenzoic acid $(12.72 \mu \mathrm{g} / \mathrm{mL})$, and low concentrations of caffeic acid $(6.15 \mu \mathrm{g} / \mathrm{mL})$, quercetin$3-\beta$-glucoside $(4.97 \mu \mathrm{g} / \mathrm{mL})$ and isorhamnetin $(4.68 \mu \mathrm{g} / \mathrm{mL})$. In the stem's extracts, the highest concentrations were of ferulic acid $(7.96 \mu \mathrm{g} / \mathrm{mL})$, rutin $(5.61 \mu \mathrm{g} / \mathrm{mL})$ and catechin $(4.37 \mu \mathrm{g} / \mathrm{mL})$; medium concentration were identified for isorhamnetin $(3.31 \mu \mathrm{g} / \mathrm{mL})$ and quercetin- $3-\beta$-glucoside $(2.01 \mu \mathrm{g} / \mathrm{mL})$ and apigenin $(0.79 \mu \mathrm{g} /$ $\mathrm{mL}$ ) was identified at the low concentrations. Gallic acid, caffeic acid or 2,5-dihydroxybenzoic acid were not detected.

Limitations/implications: Some flavonoids have been identified in other Acer species but have not been identified and quantified in Acer negundo, a Mexican species.

Findings/conclusions: For the first time we report gentisic acid in Acer negundo leaf extracts. This analytical method can be standardized to serve as a quality analysis of maple tree products.
\end{abstract}

Key words: ferulic acid, gentisic acid, flavonoids, HPLC.

\section{INTRODUCTION}

The genus Acer belongs to the family Aceraceae and the order Sapindaleae, comprising 180 species (Li et al., 2010). Maples (Acer spp.) are important in the northern hemisphere, in regions of East Asia, North America and Europe (Glensk et al., 2009). Acer negundo is used in reforestation programs. The sap is a component that acts as a sugar source. It is currently an endangered species. The reported pharmacological activities of Acer species are 
antioxidant (Zhang et al., 2014), antitumor (Kim et al., 2015), anti-inflammatory (Ko and Choi, 2015), antibacterial (Maisuria et al., 2015), antifungal, antiviral (Song et al., 2015), antihyperglycemic (Zhang et al., 2015), hepatoprotective (Yoo et al., 201 1), antiobesity (Gao et al., 2012) and osteoblastic differentiation stimulator (Ha et al., 2014). There are studies of compounds isolated from Acer showing antidepressant, skin-protective, neuroprotective, vasorelaxant, antihypertensive and antimutagenic properties. From the maple trees maple syrup is produced, an inexpensive, highly commercial product containing carbohydrates (glucose, fructose, sucrose and high molecular weight polysaccharides), minerals (Al, Ca, $\mathrm{Fe}, \mathrm{K}, \mathrm{Mg}, \mathrm{Mn}, \mathrm{Na}$, and $\mathrm{Zn}$ ), vitamins (niacin, riboflavin, and thiamine), amino acids (arginine, proline, and threonine) and organic acids (fumaric acid, and malic acid) (Zhang et al., 2014). Many highly bioactive compounds have been extracted from Acer species, such as flavonoids, tannins, alkaloids, terpenoids, and phenolic compounds such as lignans, phenolic acids, stilbenes, and coumarins.

The chemical components with the highest bioactivity extracted from the Acer genus are benzoic acid derivatives (5.4\%), diarylheptanoids (8.8\%), simple phenolic compounds (9.7\%), terpenoids and phytosterols (9.7\%), tannins (12.4\%), flavonoids (18.7\%), phenylpropanoids (22.4\%), among other compounds such as alkaloids and phenylethyl glycosides (Bi et al., 2016). The most characteristic compounds existing in Acer species are flavonoids and tannins (Liu et al., 2013). The Acer genus also contains important phytochemical compounds such as triterpene saponins (Glensk et al., 2009). Some 331 chemical compounds have been identified from 34 species of the Acer genus (Bi et al., 2016). However, most of the compounds from $A$. negundo have not yet been reported.

High Performance Liquid Chromatography (HPLG) is well suited for both qualitative and quantitative monitoring of various trees and has been widely used to evaluate tree and medicinal resources. Therefore, in the present research, HPLC analysis was used to analyze flavonoids from $A$. negundo extracts. There are research reports on the identification of flavonoids in Acer species, but do not record their quantification. Therefore, the present work reports the quantification of flavonoids obtained from renewable sources of $A$. negundo trees (leaf and stem extracts) by high performance liquid chromatography analysis, so that they are used as marker compounds for chemical evaluation or standardization of A. negundo and its products.

\section{MATERIALS AND METHODS}

Standards and reagents. The solvents used for the extraction and high performance liquid chromatography procedures were HPLC and analytical grade, respectively, and obtained from Sigma-Aldrich (St Louis, MO, USA). All stock solutions, standards, samples, solvents, and reagents were filtered through $0.20 \mu \mathrm{m}$ PTFE membrane filters (Phenomenex, USA) prior to separation or injection into the instrument.

Sample collection. Stem and whole leaves of $A$. negundo were collected during autumn (October-November) 2019 in the vicinity of the city of Morelia, State of Michoacán, Mexico (1946’ 06" N 101 11'22" W, 1920 masl).

Obtaining the extracts. Hundred $\mathrm{mg}$ samples of dried leaves and $100 \mathrm{mg}$ of dried stems of $A$. negundo were taken by triplicate, these organs are renewable sources so as not 
to damage the trees. The leaves and stems of $A$. negundo were macerated in a mortar with a pistil. In a $250 \mathrm{~mL}$ flask, $100 \mathrm{~mL}$ of $80 \%$ ethanol were added to each sample, the mouth of the flask was covered with aluminum foil, and each sample was allowed to rest for $24 \mathrm{~h}$. The samples were then filtered on filter paper. Finally, a rotary evaporator (Buchi brand) was used to evaporate the solvent from each extract. The crude extracts were placed in amber bottles for further analysis.

HPLC analysis. The profile of phenolic compounds was determined from the methods modified by Espinosa-Alonso et al. (2006) and Valdez-Morales et al. (2014), with some modifications. Flavonoids and phenolic compounds were identified and quantified on an automatic injection chromatograph model Ultimate 3000, Dionex brand, equipped with a quaternary pump and a diode array detector. An Acclaim 120, C18 column (4.6 mm $\times 250 \mathrm{~mm}, 5$ microns, Thermo Sci brand) was used. The mobile phase used consisted of $\mathrm{A}=$ acidified water to $\mathrm{pH} 2.8$ with acetic acid and $\mathrm{B}=$ acetonitrile, a gradient was used starting with $90 \% \mathrm{~A}$ and $10 \% \mathrm{~B}$ up to $2.5 \mathrm{~min}$, gradually increasing the percentage of $\mathrm{B}$ : $12 \%$ at $6 \mathrm{~min}, 23 \%$ at $18 \mathrm{~min}, 35 \%$ at $24 \mathrm{~min}, 95 \%$ at $30 \mathrm{~min}$ and returning to the initial conditions of $90 \% \mathrm{~A}$ in a final time of $40 \mathrm{~min}$.

The rest of the chromatographic conditions are summarized: flow rate of $0.3 \mathrm{~mL} /$ min, injection volume of $10 \mu \mathrm{L}$, and the recorded wavelengths were 260, 280, 300, 320, 350, $375 \mathrm{~nm}$. The compounds were identified by comparing their retention times and absorption spectra with the previously run standards and with which the calibration curves were made. The Chromeleon 7.0 software was used for the chromatographic analysis. The concentration values of each phenolic compound were calculated from the area under the signal curve observed in the chromatogram.

Compounds were identified as a function of their retention time and absorption spectra. Identity was only assigned to signals with a purity greater than 980 (1000 being the maximum value). The areas at the wavelength of maximum absorption of each compound were captured, as was the corresponding standard curve used. Flavonoid content was analyzed with a mean comparison test ANOVA $(\mathrm{P}<0.05)$, in the SAS statistical software (v. 2018).

\section{RESULTS AND DISGUSSION}

HPLC analysis. The isolated flavonoids from $A$. negundo extracts and identified by chromatographic analysis are shown in the chromatograms in Figure 1 and 2.

The Acer genus is characterized by the biosynthesis of phenolic compounds such as lignans, phenolic acids, stilbenes, coumarins and various flavonoid subclasses. Thirty-one phenolic compounds have been reported for the genus Acer (Bi et al., 2016). The phenolic group in flavonoids directly acts by capturing missing electrons from Reactive Oxygen Species (ROS), generating less reactive species. Flavonoids act as buffers, capturing free radicals that generate the less reactive flavin radical, as the missing electrons are delocalized in it. Flavonoids can prevent the cancer occurrence by acting as natural antioxidants that prevent damage to cellular DNA caused by ROS or carcinogens. Flavonoids, both glucosides and glycosides, are also important antioxidant compounds (Wijeratne et al., 2006). Secondary plant compounds, such as flavonoids 
and other phenylpropanoid derivatives, act as attractants and deterrents to potential insect herbivores (Harborne and Williams, 2000). Phytochemical studies have also been conducted to investigate the phytochemical content in Acer species wood. Japanese maple bark has been investigated for its anticancer, anti-inflammatory, antifungal, and antibacterial effects. The bark is used in traditional Japanese medicine to treat liver disorders. Compounds isolated from A. nikoenses bark were catechin, rhododendrol, centrolobol, acerogenin A, B, D, K, and M, and acerides I, II, and IV (Li and Seeram $e t$ al., 2011).

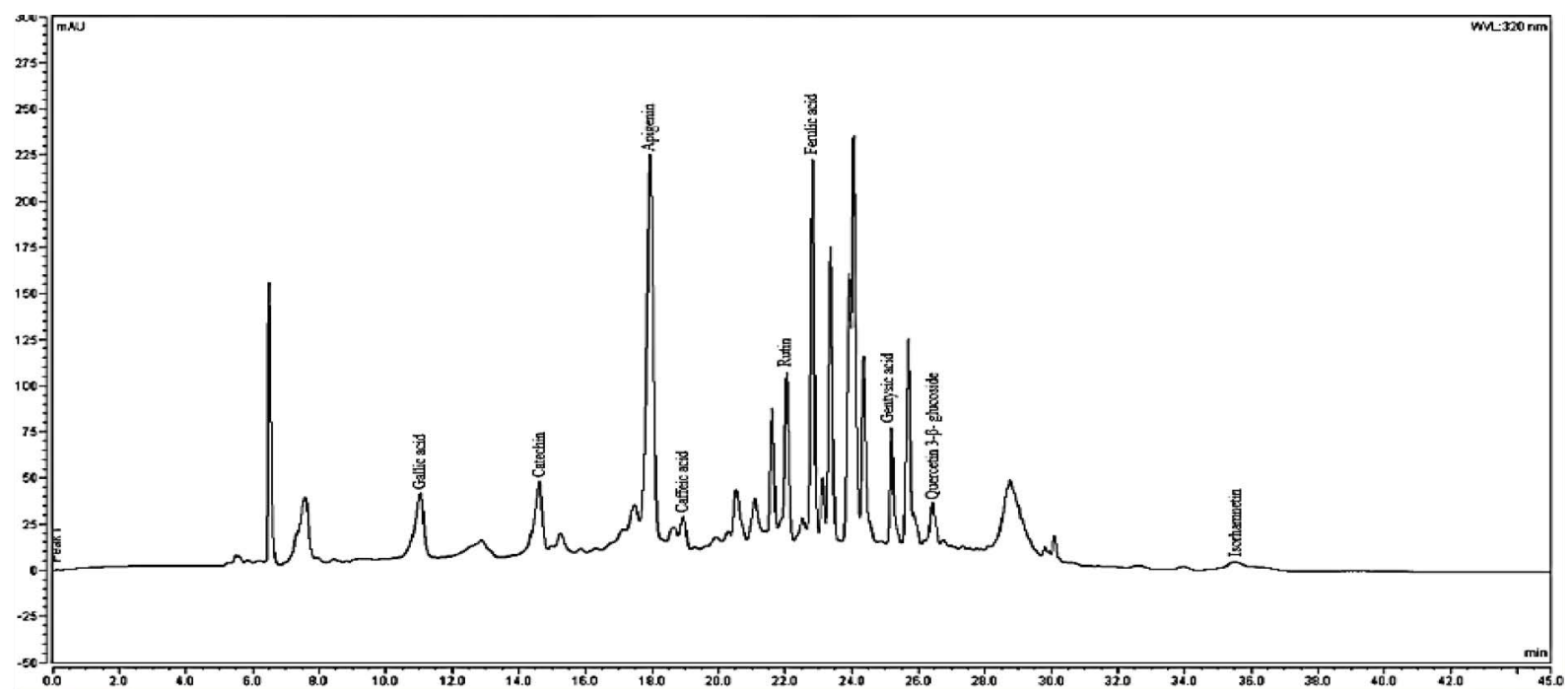

Figure 1. Compound identification by HPLC from leaves of $A$. negundo. Chromatograms of flavonoids, which were obtained from ethanol extract of $A$. negundo by column chromatography. Gallic acid $\left(\mathrm{C}_{7} \mathrm{H}_{6} \mathrm{O}_{5}\right)$, catechin $\left(\mathrm{C}_{15} \mathrm{H}_{14} \mathrm{O}_{6}\right)$, apigenin $\left(\mathrm{C}_{15} \mathrm{H}_{10} \mathrm{O}_{5}\right)$, caffeic acid $\left(\mathrm{C}_{9} \mathrm{H}_{8} \mathrm{O}_{4}\right)$, rutin $\left(\mathrm{C}_{27} \mathrm{H}_{30} \mathrm{O}_{16}\right)$, ferulic acid $\left(\mathrm{C}_{10} \mathrm{H}_{10} \mathrm{O}_{4}\right)$, gentisic acid (2,5-dihydroxybenzoic acid; $\left.\mathrm{C}_{7} \mathrm{H}_{6} \mathrm{O}_{4}\right)$, quercetin 3 - $\beta$-glucoside $\left(\mathrm{C}_{21} \mathrm{H}_{20} \mathrm{O}_{12}\right)$, and isorhamnetin $\left(\mathrm{C}_{16} \mathrm{H}_{12} \mathrm{O}_{7}\right)$.

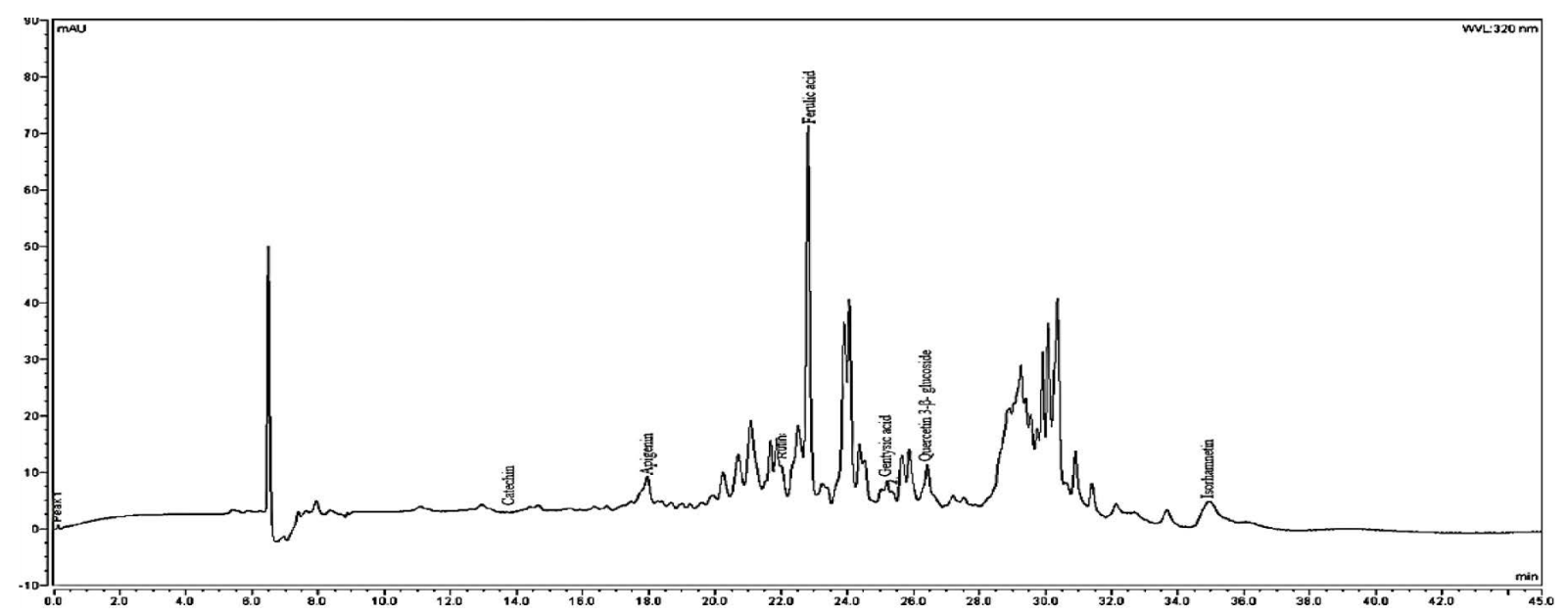

Figure 2. Compound identification by HPLC in A. negundo stems. Chromatograms of flavonoids, which were obtained from the ethanol extract of $A$. negundo by column chromatography. Catechin $\left(\mathrm{C}_{15} \mathrm{H}_{14} \mathrm{O}_{6}\right)$, apigenin $\left(\mathrm{C}_{15} \mathrm{H}_{10} \mathrm{O}_{5}\right)$, rutin $\left(\mathrm{C}_{27} \mathrm{H}_{30} \mathrm{O}_{16}\right)$, ferulic acid $\left(\mathrm{C}_{10} \mathrm{H}_{10} \mathrm{O}_{4}\right)$, quercetin 3- $\beta$-glucoside $\left(\mathrm{C}_{21} \mathrm{H}_{20} \mathrm{O}_{12}\right)$, and isorhamnetin $\left(\mathrm{C}_{16} \mathrm{H}_{12} \mathrm{O}_{7}\right)$. 
Understanding the differences between the compounds of different species of the Acer genus brings us closer to the chemotaxonomic distribution of the compounds. In $A$. campestre wood, cellulose, pentosan/xylan and lignin contents were determined with HPLC (Antczak et al. 2013).

1) Gallic acid. Zhang et al. (2015) identified gallic acid in leaf extracts of $A$.pseudoplatanus by nuclear magnetic resonance. Gallic acid (tannin) is also present in the stem, leaf and bark of A. barbinerve, A. tataricum, A. negundo, A. platanoides, A. rubrum, A. truncatum, A. pentapomicum (Dong et al., 2006). In addition, many galloyl-containing flavonoid glycosides have been isolated from the leaves of A. tataricum subsp. ginnala (Maxim.) Wesm., Acer okamotoanum, A. rubrum L. and A. platanoides L., and two glycosides exhibited strong inhibitory activity against HIV-1 integrase (Kim et al., 1998). Among these flavonoids, anthocyanins have been of great interest because they are responsible for the leaf color change in spring and autumn. Cyanidin-3-(2",3"-digalloyl- $\beta$-glucopyranoside) was the first example of a di-acetylated anthocyanin with gallic acid (Fossen and Andersen 1999). In our research we found gallic acid in leaf extracts $(19.04 \mu \mathrm{g} / \mathrm{mL})$ but not in stem extracts.

2) Catechin. Nugroho et al. (2015) analyzed three phenolic substances (salidroside, catechin and scopoletin) by HPLC analysis from three methanolic extracts of stem bark, heartwood and leaves of $A$. tegmentosum. The major metabolite produced from the methanolic extract of stem bark was: salidroside: $80.22 \mathrm{mg} / \mathrm{g}$, and in lower concentration catechin: $23.31 \mathrm{mg} / \mathrm{g}$, and scopoletin $9.45 \mathrm{mg} / \mathrm{g}$. Catechin was identified in extracts of stems, stem bark, wood and bark of $A$. barbinerve, A. mandshuricum, A. maximowiczianum, $A$. rubrum, A. tegmentosum (Lee et al., 2014). In our research, we found catechin in leaves (33.97 $\mu \mathrm{g} / \mathrm{mL})$ and stems $(4.37 \mu \mathrm{g} / \mathrm{mL})$ of $A$. negundo extracts.

3) Apigenin. In leaf extracts of A. palmatum (Aritomi, 1963) and A. oblongum (Parveen et al., 1988) the flavonoid apigenin was found. In our research, we found apigenin in the extracts of leaves $(19.05 \mu \mathrm{g} / \mathrm{mL})$ and stems $(0.79 \mu \mathrm{g} / \mathrm{mL})$ of $A$. negundo.

4) Caffeic acid. Caffeic acid was identified in dormant shoots of $A$. saccharum (Thakur, 1977). Here, caffeic acid was identified in $A$. negundo leaf extracts $(6.15 \mu \mathrm{g} / \mathrm{mL})$ but not in stem extracts.

5) Rutin. The flavonoid rutin was found in both, leaves and stem bark of $A$. tataricum subsp. ginnala, A. glabrum, A. rubrum and A. negundo (Backheet, 2003). In our study we found rutin in the leaf $(34.19 \mu \mathrm{g} / \mathrm{mL})$ and stem $(5.61 \mu \mathrm{g} / \mathrm{mL})$ extracts of $A$. negundo.

6) Ferulic acid. In dormant shoots of $A$. saccharum they ferulic acid has been identified (Thakur, 1977). In this research we found ferulic acid, a phenylpropanoid, in extracts from the leaves $(17.2 \mu \mathrm{g} / \mathrm{mL})$ and stems $(7.96 \mu \mathrm{g} / \mathrm{mL})$ of $A$. negundo.

7) Gentisic acid (2,5-dihydroxybenzoic acid). As hydroquinone, gentisic acid easily oxidizes and is used as an antioxidant excipient in some pharmaceutical preparations (Kostiuk et al., 1988). In this research, ferulic acid was identified in A. negundo leaf extracts $(12.72 \mu \mathrm{g} / \mathrm{mL})$ but not present in stem extracts.

8) Quercetin 3- $\beta$-glucoside. Quercetin has anti-ulcer properties by protecting the gastric mucosa (de Lira et al., 2009). Ma et al. (2005) isolated quercetin-3-O-L-rhamnoside from A. truncatum by HSCCG (High-Speed Counter-Current Chromatography) type chromatography, the analysis was based on studying an ethyl acetate extract of the leaves. 
We obtained $41.9 \mathrm{mg}$ of quercetin-3-O-L-rhamnoside from $366 \mathrm{mg}$ of the crude extract. In our research, we found quercetin-3- $\beta$-glucoside in leaf extracts $(4.97 \mu \mathrm{g} / \mathrm{mL})$ and stem extracts $(2.01 \mu \mathrm{g} / \mathrm{mL})$ of $A$. negundo.

9) Isorhamnetin. Isorhamnetin was found in the leaves of A. glabrum (Justice et al., 1995). Isorhamnetin-3-O-ruthinoside has also been identified (Backheet, 2003). In our study we identified isorhamnetin in leaf extracts $(4.68 \mu \mathrm{g} / \mathrm{mL})$ and stem extracts $(3.31 \mu \mathrm{g} /$ $\mathrm{mL}$ ) of $A$. negundo. Glensk et al. (2009) identified a triterpene saponin from A. velutinum leaf extracts by NMR spectroscopy, $21 \beta$-saponin, $22 \alpha$-O-diangeloylprotoaescigenin, and it exhibited in vitro cytotoxic activity against HL-60, B16-F0 and BALB/3T3 cell lines. Also, several authors have analyzed the flavonoid content in maple syrup. Ann (2013) analyzed four grades of maple syrup (extra light, light, medium, and dark) from the 2007 harvest. Twenty-four phenolic compounds were isolated from medium grade syrup and identified by spectral and chemical tests. They were found to have: (a) benzoic acid and several hydroxylated and methoxylated derivatives (gallic acid, 1-O-galloyl- $\beta$-d-glucose, and $\gamma$-resorcylic acid); (b) cinnamic acid derivatives, coumaric acid, 4-methoxycinnamic acid, caffeic acid, ferulic acid, sinaptic acid, and chlorogenic acid ester); (c) flavonoids, flavanols, catechin and epicatechin, and flavonols of kaempferol and its 3 - $\mathrm{O}-\beta$-d-glucoside, 3-O- $\beta$-d-galactoside, quercetin and its $3-\mathrm{O}-\beta$-d-glucoside, $3-\mathrm{O}-\beta$-L-rhamnoside and 3-O-rhamnoglucoside (rutin). Traces obtained at 280 and $350 \mathrm{~nm}$ in the HPLC series of the ethyl acetate soluble fractions of eight samples indicated the presence of several phenolic substances, mostly at very low concentration with some variability in peak heights, but not in retention times, among the syrups.

Authors such as Geoffroy et al. (2019) studied hot water extracts of A. saccharum bark and shoots, proving that they contain large amounts of phenolic structures that can be used as antioxidant food additives. By performing a replication based on high Performance Liquid Chromatography-High Performance Liquid Chromatography-High Performance Mass Spectrometry (HPLC-DAD-HRMS), it has been showed that hot water extracts of A. saccharum bark are rich in simple phenolic compounds and phenylpropanoid derivatives, while the extract of shoots predominantly contains flavonoids, benzoic acids and their complex derivatives, such as condensed and hydrolyzable tannins (Geoffroy et al., 2019).

\section{GONGLUSIONS}

Our research revealed that leaf extracts contained a large number of flavonoids compared to stem extracts. A. negundo leaf extracts had higher rutin and catechin concentrations; in intermediate concentration were apigenin, gallic acid, ferulic acid, and 2,5-dihydroxybenzoic acid; and in the lowest concentration caffeic acid, quercetin-3- $\beta$ glucoside, and isorhamnetin. In A. negundo stem extracts there was a higher concentration of ferulic acid, rutin and catechin; in medium concentration were isorhamnetin and quercetin-3- $\beta$-glucoside and in the lowest concentration apigenin, but no gallic acid presence, caffeic acid or 2,5-dihydroxybenzoic acid. We report for the first time the presence of gentisic acid in $A$. negundo leaf extracts but not in stem extracts. The method developed to characterize $A$. negundo leaves and stems is rapid and highly sensitive using HPLC. This analytical method can be standardized to serve as a quality analysis for maple 
products. With the increasing commercial demand for natural products, phenolic profiles of leaf and stem extracts will help promote these Acer negundo derivatives as new sources of bioactive compounds for the food, nutraceutical, and cosmetic industries.

\section{ACKNOWLEDGMENTS}

The corresponding author thanks the support of the Consejo Nacional de Ciencia y Tecnología (CONACyT)México and the Universidad Michoacana de San Nicolás de Hidalgo, Morelia.

\section{REFERENGES}

Antczak, A., Michaluszko, A., Klosinska, T., \& Drozdzek, M. (2013). Determination of the Structural Substances Content in the Field Maple Wood (Acer Campestre L.) - Comparison of The Classical Methods with Instrumental. Biol Pharm Bull (82): pp.11-17.

Aritomi, M. (1963). Chemical Constituents in Aceraceous Plants. I. Flavonoid Constituents in The Leaves of Acer palmatum Thunberg. Yakugaku Zasshi (83): pp.737-740.

Backheet, E.Y. (2003). Gallotannin and Flavonoid Glycosides from the Stem Bark of Acer Negundo (L.). Biol Pharm Bull 26: pp.77-82.

Bi, W., Gao, Y., Shen, J., He, G., Liu, H., Peng, Y., Zhang, C., \& Xiao, P. (2016). Traditional Uses, Phytochemistry, and Pharmacology of the Genus Acer (Maple): A Review. J Ethnopharmacol 189: pp.31-60. Doi: 10.1016/j.jep.2016.04.021

De Lira, M.K.S., Nunes, D.G.E., Ferreira, P.M.E., Luiz-Ferreira, A., Souza-Brito, A.R.M., Hiruma-Lima, C.A., Barbosa-Filho, J.M., \& Batista, L.M. (2009). Flavonoids with Gastoprotective Activity. Molecules 14: pp.979-1012. Doi:10.3390/molecules14030979

Dong, L.P., Liu, H.Y., \& Ni, W. (2006). Four New Compounds from the Leaves of Acer Truncatum. Chem Biodivers 3 (7): pp.791-798. Doi: 10.1002/cbdv.200690081

Espinosa-Alonso, L., Lygin, A., Widholm,J., Valverde, M., \& Paredes-López, O. (2006). Polyphenols in Wild and Weedy Mexican Common Beans (Phaseolus vulgaris L.). J Agric Food Chem 54 (12): pp.4436-4444. Doi: 10.1021/jf060185e

Fossen, T., \& Andersen, M. (1999). Cyanidin 3-(2", 3"-Digalloylglucoside) from Red Leaves of Acer platanoides. Phytochem 52: pp.1697-1700. Doi:10.1016/S0031-9422(99)00188-0

Gao, L., Cao, L., Tian, M., \& Chen, Z. (2012). Study on The Weight-Reducing Effect of Acer Truncatum Leave Extract in Alimentary Obesity Rat. J Hyg Res 41 (4): pp.609-611.

Geoffroy, T.R., Stevanovic, T., Fortin, Y., Poubelle, P.E., \& Meda, N. (2019). Metabolite Profiling of Two Maple-Derived Products Using Dereplication Based on High-Performance Liquid Chromatography-Diode Array Detector-Electrospray Ionization-Time-of-Flight-Mass Spectrometry: Sugar Maple Bark and Bud Hot-Water Extracts. J Agric Food Chem 67 (32): pp.8819-8838. Doi: 10.1021/acs.jafc.9b02664

Ha, H., Shim, K.S., Kim, T., An, H., Lee, C.J., Lee, K.J., \& Ma, J.Y. (2014). Water Extract of Acer Tegmentosum Reduces Bone Destruction by Inhibiting Osteoclast Differentiation and Function. Molecules 19(4) : pp.3940-3954. Doi: 10.3390/molecules 19043940

Harborne, J.B., \& Williams, C.A. (2000). Advances in Flavonoid Research Since 1992. Phytochem 55 (6): pp.481-504. Doi: 10.1016/S00319422(00)00235-1

Justice, D.E., Reid, A.R., \& Bohm, B.A. (1995). Vacuolar Flavonoids of Rocky-Mountain Maple, Acer glabrum Torrey (Aceraceae). Biochem Syst Ecol 23 (3):pp. 263-265. Doi: 10.1016/0305-1978(95)00014-L

Kim, I.W., Jeong, H.S., Kim, J.K., Lee, J.K., Kim, H.R., Yun, H.Y., Baek, K.J., Kwon, N.S., Park, K.C., \& Kim, D.S. (2015). Methyl Gallate from Acer Barbinerve Decreases Melanin Synthesis in Mel-Ab Cell. Die Pharmazie 70 (1): pp.55-59. Doi: 10.1691/ph.2015.4683

Kim, H.J., Woo, E.R., \& Shin, G.G. (1998). A New Flavonol Glycoside Gallate Ester from Acer Okamotoanum and its Inhibitory Activity Against Human Immunodeficiency Virus-1 (HIV-1) Integrase. J Nat Prod 61(1) : pp.145-148. Doi: 10.1021/np970171q

Ko, E.K., \& Choi, S.E. (2015). Inhibitory Effects of Phenolic Compounds from Stems of Acer ginnala on Nitric Oxide Production. J Chem Pharm Res 7 (2): pp.395-402.

Kostiuk, V.A., Potapovich, A.I., Tereshchenko, S.M., \& Afanasev, I.B. (1988). Antioxidant Activity of Flavonoids in Various Systems of Lipid Peroxidation. Biokhimiia 53 (8): pp.1365-1370.

Lee, K.J., Song, N.Y., Oh, Y.C., Cho, W.K., \& Ma, J.Y. (2014). Isolation and Bioactivity Analysis of Ethyl Acetate Extract from Acer Tegmentosum Using In Vitro Assay and On-Line Screening HPLC-ABTS (+) System. J Anal Methods Chem 2014.pp.1-15. Doi: 10.1155/2014/150509.

Li, L., \& Seeram, N.P. (201 1). Further Investigation into Maple Syrup Yields 3 New Lignans, a New Phenylpropanoid, and 26 Other Phytochemicals. J Agric Food Chem 59(14) : pp.7708-7716. doi: 10.1021/jf2011613.

Li, L.Y., \& Seeram, N.P. (2010). Maple Syrup Phytochemicals Include Lignans, Coumarins, a Stilbene, and Other Previously Unreported Antioxidant Phenolic Compounds. J Agric Food Chem 58 (22): pp.11673-11679. doi: 10.1021/jf1033398.

Liu, W., Ouyang, Y., \& Wan, C.P. (2013). Flavonoids of the Genus Acer. Asian J Chem 25 (13): pp.7075-7078. doi: 10.14233/ajchem.2013.14643

Ma, X., Tian, W., Wu, L., Cao, X., \& Ito, Y. (2005). Isolation of Quercetin-3-O-L-Rhamnoside from Acer truncatum Bunge by High-Speed Counter-Current Chromatography. J Chromatogr A 1070: 211-214. doi:10.1016/j.chroma.2005.02.052.

Maisuria, V.B., Hosseinidoust, Z., \& Tufenkji, N. (2015). Polyphenolic Extract from Maple Syrup Potentiates Antibiotic Susceptibility and Reduces Biofilm Formation of Pathogenic Bacteria. Appl Environ Microbiol 81 (11): pp.3782-3792. doi: 10.1128/AEM.00239-15

Glensk, M., Włodarczyk, M., Bassarello, G., Pizza, C., Stefanowicz, P., \& Switalska, M. (2009). A Major Saponin from Leaves Extract of Acer velutinum. Zeitschrift für Naturforschung 64 (9): pp.1081-1086. doi: 10.1515/znb-2009-0915 
Nugroho, A., Song, B.M., \& Park, H.J. (2015). HPLC and GC-MS Analysis of Phenolic Substances in Acer tegmentosum. Nat Prod Sci 21 (2): pp.8792. Doi: $10.0000 /$ nps.2015.21.2.87.

Parveen, N., Khan, N.U., \& Inoue, T. (1988). Ethyl Brevifolin Carboxylate and Other Constituents from Acer oblongum Leaves. Phytochem 27 (12): pp.3990-3991.Doi: 10.1016/0031-9422(88)83068-1

Song, J.H., Park, K., Shim, A., Kwon, B.E., Ahn, J.H., Choi, Y.J., Kim, J.K., Yeo, S.G., Yoon, K., \& Ko, H.J. (2015). Complete Sequence Analysis and Antiviral Screening of Medicinal Plants for Human Coxsackievirus A16 Isolated in Korea. Osong Public Health Res Perspect 6 (1): pp.52-58. Doi: 10.1016/j.phrp.2014.12.004

Thakur, M.L. (1977). Phenolic Growth Inhibitors Isolated from Dormant Buds of Sugar Maple (Acer saccharum Marsh). J Exp Bot 28 (4): pp.795803. doi: $10.1093 / \mathrm{jxb} / 28.4 .795$

Valdez-Morales, M., Espinosa-Alonso, L.G., Espinoza-Torres, L.C., Delgado-Vargas, F., \& Medina-Godoy, S. (2014). Phenolic Content and Antioxidant and Antimutagenic Activities in Tomato Peel, Seeds, and Byproducts. J Agric Food Chem 62 (23): pp.5281-5289. doi: $10.1021 / \mathrm{j} f 5012374$

Wijeratne, S.S.K., Abou-Zaid, M.M., \& Shahidi, F. (2006). Antioxidant Polyphenols in Almond and its Coproducts. J Agric Food Chem 54 (2): pp.312-318. doi: 10.1021/jf051692j

Zhang, L., Tu, Z.C., Yuan, T., Ma, H., Niesen, D.B., Wang, H., \& Seeram, N.P. (2015). New Gallotannin and Other Phytochemicals from Sycamore Maple (Acer pseudoplatanus) Leaves. Nat Prod Commun 10 (11): pp.1977-1980. doi: 10.1177/1934578X1501001143

Yoo, Y.M., Joung, E.M., Kang, H.Y., Choi, I.G., Choi, K.C., \& Jeung, E.B. (2011). The Sap of Acer okamotoanum Decreases Serum Alcohol Levels After Acute Ethanol Ingestion in Rats. Int J Mol Med 28 (4): pp.489-495. doi: 10.3892/ijmm.2011.724

Zhang, Y., Yuan, T., Li, L.Y., Nahar, P., Slitt, A., \& Seeram, N.P. (2014). Chemical Compositional, Biological, and Safety Studies of a Novel Maple Syrup Derived Extract for Nutraceutical Applications. J Agric Food Chem 62 (28): 6687-6698. doi: 10.1021/jf501924y 